\title{
RESEARCH
}

Open Access

\section{The relationship between physical activity, apolipoprotein E \&4 carriage, and brain health}

Jaisalmer de Frutos-Lucas ${ }^{1,2,3^{*}}$, Pablo Cuesta ${ }^{2,4}$, David López-Sanz ${ }^{2,5}$, África Peral-Suárez ${ }^{6}$, Esther Cuadrado-Soto ${ }^{6}$, Federico Ramírez-Toraño ${ }^{2,7}$, Belinda M. Brown' ${ }^{8}$, Juan M. Serrano', Simon M. Laws ${ }^{3,9}$, Inmaculada C. Rodríguez-Rojo 2,10, Juan Verdejo-Román 2,7,11, Ricardo Bruña 2,7,12, Maria L. Delgado-Losada7, Ana Barabash ${ }^{13,14}$, Ana M. López-Sobaler ${ }^{6}$, Ramón López-Higes ${ }^{7}$, Alberto Marcos ${ }^{15}$ and Fernando Maestú $2,7,12$

\begin{abstract}
Background: Neuronal hyperexcitability and hypersynchrony have been described as key features of neurophysiological dysfunctions in the Alzheimer's disease (AD) continuum. Conversely, physical activity (PA) has been associated with improved brain health and reduced AD risk. However, there is controversy regarding whether $\mathrm{AD}$ genetic risk (in terms of $A P O E \varepsilon 4$ carriage) modulates these relationships. The utilization of multiple outcome measures within one sample may strengthen our understanding of this complex phenomenon.

Method: The relationship between PA and functional connectivity (FC) was examined in a sample of 107 healthy older adults using magnetoencephalography. Additionally, we explored whether $\varepsilon 4$ carriage modulates this association. The correlation between FC and brain structural integrity, cognition, and mood was also investigated.

Results: A relationship between higher PA and decreased FC (hyposynchrony) in the left temporal lobe was observed among all individuals (across the whole sample, in $\varepsilon 4$ carriers, and in $\varepsilon 4$ non-carriers), but its effects manifest differently according to genetic risk. In $\varepsilon 4$ carriers, we report an association between this region-specific FC profile and preserved brain structure (greater gray matter volumes and higher integrity of white matter tracts). In this group, decreased FC also correlated with reduced anxiety levels. In $\varepsilon 4$ non-carriers, this profile is associated with improved cognition (working and episodic memory).
\end{abstract}

Conclusions: PA could mitigate the increase in FC (hypersynchronization) that characterizes preclinical AD, being beneficial for all individuals, especially $\varepsilon 4$ carriers.

Keywords: Physical activity, APOE, MEG, Synaptic function, Alzheimer's disease, Temporal lobe

\footnotetext{
* Correspondence: jaisalmer.defrutos@ctb.upm.es

'Biological and Health Psychology Department, School of Psychology,

Universidad Autonoma de Madrid, 28049 Madrid, Spain

${ }^{2}$ Laboratory of Cognitive and Computational Neuroscience (UCM-UPM),

Center for biomedical Technology, Parque Científico y Tecnológico de la

UPM, Crta. M40, Km. 38, 28223 Pozuelo de Alarcón, Madrid, Spain

Full list of author information is available at the end of the article
}

(c) The Author(s). 2020 Open Access This article is licensed under a Creative Commons Attribution 4.0 International License, which permits use, sharing, adaptation, distribution and reproduction in any medium or format, as long as you give appropriate credit to the original author(s) and the source, provide a link to the Creative Commons licence, and indicate if changes were made. The images or other third party material in this article are included in the article's Creative Commons licence, unless indicated otherwise in a credit line to the material. If material is not included in the article's Creative Commons licence and your intended use is not permitted by statutory regulation or exceeds the permitted use, you will need to obtain permission directly from the copyright holder. To view a copy of this licence, visit http://creativecommons.org/licenses/by/4.0/. The Creative Commons Public Domain Dedication waiver (http://creativecommons.org/publicdomain/zero/1.0/) applies to the data made available in this article, unless otherwise stated in a credit line to the data. 


\section{Background}

Physical activity (PA) has been persistently referred to as the twenty-first century panacea. In both clinical and non-clinical populations, PA is related to improvements in sleep quality, mood, cognitive performance, and perceived quality of life [1]. In addition, PA is associated with marked decreases in the risk of a broad spectrum of diseases, including diabetes mellitus, cancer, and dementia [1]. In the specific case of Alzheimer's disease (AD; the most common form of dementia), PA has been found to reduce incidence, AD-associated neuropathological burden, and cognitive decline [2-4].

The major genetic risk factor for sporadic $A D$, namely the apolipoprotein $\mathrm{E}(A P O E) \quad \varepsilon 4$ allele, is present in $60-80 \%$ of $\mathrm{AD}$ cases and is linked to a 3.2-fold increased AD risk in heterozygosis and up to 14.9-fold increased AD risk in homozygosis [5]. While most studies claim that PA-protective effects mainly manifest among $\varepsilon 4$ carriers [6, 7], others identified this same effect exists only in non-carriers [8, 9], while other studies report benefits regardless of $A P O E$ $\varepsilon 4$ allele carriage $[10,11]$. This inconsistent literature is likely due to the use of varying study designs and outcome measures (i.e., hippocampal volume, shortterm memory, or rate of $\mathrm{AD}$ conversion). To further understand the modulating effect of $\mathrm{AD}$ genetic risk on the relationship between PA and brain health, a more detailed investigation using varied outcome measures is warranted.

Based on the inconsistencies in previous literature, the current study will first investigate the relationship between PA and synaptic activity in cognitively healthy older adults. Synaptic activity will be captured employing magnetoencephalography and analyzed under the framework of network synchronization. It is believed that the flow of information between different brain regions is sustained by synchronous changes in the frequency, pattern, or strength of their oscillatory activity [12]. Early loss of inhibitory neurons in preclinical AD leads to a state of increased hyperexcitability and hypersynchrony [13-15], which has been found to augment amyloid release and produce neurotoxic effects [16, 17]. We hypothesize that PA exerts a neuroprotective effect that will be associated with reduced network synchrony in both groups, in opposition to the state of synaptic hyperexcitability that characterizes preclinical and prodromal AD [18-22]. Then, once we have identified the functional connectivity (FC) patterns that are influenced by PA level, we will explore if there are any associations between these FC patterns and structural integrity (gray and white matter), cognition, and mood. Additionally, we will examine if $A P O E \& 4$ allele carriage modulates these relationships.

\section{Methods and materials \\ Participants}

A total of 262 individuals participated in a study aimed to characterize the neurophysiological features of healthy aging. Participants were recruited from local hospitals and through several dissemination talks, and a team of expert neuropsychologists assessed that they met inclusion criteria. A detailed list of exclusion criteria can be found in [23]. The procedure was performed following current guidelines and regulations, and the study was approved by the Hospital Universitario San Carlos Ethics Committee. Every participant signed an informed consent.

We included participants who had available data regarding our main variables of interest $(n=158$; MiniMental State Examination (MMSE) score, genetic information, and validated magnetic resonance imaging (MRI), MEG, and actigraphy data). We then excluded anyone with an MMSE score less than $26(n=5)$, aged less than 50 years $(n=8)$, and participants carrying less frequent $\mathrm{APOE}$ genotypes $(\varepsilon 2 \varepsilon 3, n=11 ; \varepsilon 2 \varepsilon 4, n=1$; $\varepsilon 4 \varepsilon 4, n=7$; there were no $\varepsilon 2 \varepsilon 2$ homozygotes in the original cohort). We focused on the comparison between individuals at standard genetic risk for $\mathrm{AD}(\varepsilon 3 \varepsilon 3$; hereafter non-carriers) and individuals at increased genetic risk for $\mathrm{AD}$ in heterozygosis ( $\varepsilon 3 \varepsilon 4$; hereafter; carriers) since sample sizes were insufficient to separately study the effects $\varepsilon 2$ carriage (linked to reduced risk of AD but increased risk of type III hyperlipoproteinemia [24]) and $\varepsilon 4$ carriage in homozygosis. Nevertheless, excluded genotypes are known to alter molecular and cellular dynamics [24, 25], which could potentially interfere with the neurophysiological response to exercise, and therefore, we decided not to group together all $\varepsilon 4$-carrying $(\varepsilon 2 \varepsilon 4, \varepsilon 3 \varepsilon 4, \varepsilon 4 \varepsilon 4)$ and all $\varepsilon 4$-non-carrying $(\varepsilon 2 \varepsilon 2, \varepsilon 2 \varepsilon 3$, $\varepsilon 3 \varepsilon 3)$ genotypes. Among the remaining 127 participants, there were $36 A P O E \varepsilon 4$ carriers and 91 non-carriers. We carefully selected $33 A P O E \& 4$ carriers and 74 noncarriers so that both subsamples would match in PA levels (TPA and MVPA), age, sex, educational level, MMSE, and body mass index. There were two main reasons to match the sample according to all these relevant variables instead of using them as covariates in subsequent analyses. First, including several covariates in a cluster-based permutation test could have introduced a methodological pitfall in the permutation procedure. Second, using covariates only controls for linear influences on the data, dismissing any other possible nonlinear confound.

The final sample was composed of 107 healthy older adults, aged 50-82 years. A detailed list of the sample characteristics can be found in Table 1, including scores extracted from the neuropsychological tests: Geriatric Depression Scale [26], the anxiety subscale from the Goldberg Anxiety and Depression Inventory [27], and 
Table 1 Descriptive measures of the final sample

\begin{tabular}{|c|c|c|c|c|}
\hline Variable & Whole sample & Carriers & Non-carriers & $p$ value \\
\hline $\operatorname{Sex}(M ; F)$ & $32 ; 75$ & $8 ; 25$ & $24 ; 50$ & 0.49 \\
\hline Age & $60.48 \pm 8.10$ & $59.36 \pm 7.46$ & $60.97 \pm 8.36$ & 0.39 \\
\hline Education & $4.62 \pm 0.68$ & $4.61 \pm 0.75$ & $4.622 \pm 0.63$ & 0.86 \\
\hline MMSE & $29.26 \pm 0.84$ & $29.33 \pm 0.89$ & $29.23 \pm 0.82$ & 0.42 \\
\hline BMl & $25.03 \pm 3.61$ & $25.01 \pm 3.82$ & $25.04 \pm 3.55$ & 0.90 \\
\hline TPA & $0.0125 \pm 0.0117$ & $0.0109 \pm 0.0107$ & $0.0133 \pm 0.0122$ & 0.48 \\
\hline MVPA & $36.34 \pm 21.12$ & $33.12 \pm 17.64$ & $37.77 \pm 22.47$ & 0.54 \\
\hline Forward digits & $5.95 \pm 1.22$ & $5.88 \pm 1.22$ & $5.99+1.23$ & 0.75 \\
\hline Reverse digits & $4.50 \pm 1.36$ & $4.67 \pm 1.65$ & $4.43 \pm 1.22$ & 0.55 \\
\hline Logical mem. units & $18.79 \pm 11.13$ & $20.97 \pm 11.87$ & $17.86 \pm 10.76$ & 0.25 \\
\hline Logical mem. gist & $15.74 \pm 10.87$ & $13.69 \pm 8.14$ & $16.63 \pm 11.80$ & 0.52 \\
\hline Anxiety & $1.71 \pm 0.84$ & $1.86 \pm 2.37$ & $1.65 \pm 2.05$ & 0.84 \\
\hline Depression & $1.24 \pm 1.62$ & $1.27 \pm 1.44$ & $1.23 \pm 1.70$ & 0.70 \\
\hline Total GM & $545,939 \pm 51,206$ & $546,825 \pm 66,891$ & $545,538 \pm 42,833$ & 0.80 \\
\hline Hippocampus & $7549 \pm 834$ & $7583 \pm 954$ & $7533 \pm 780$ & 0.81 \\
\hline Amygdala & $2730 \pm 470$ & $2721 \pm 573$ & $2734 \pm 420$ & 0.51 \\
\hline Precuneus & $16,337 \pm 2002$ & $16,403 \pm 2491$ & $16,306.83 \pm 1757$ & 0.87 \\
\hline Global FA & $0.4355 \pm 0.017362$ & $0.4395 \pm 0.01645$ & $0.4336 \pm 0.0176$ & 0.11 \\
\hline Uncinate & $0.4378 \pm 0.0238$ & $0.4387 \pm 0.0232$ & $0.4374 \pm 0,0242$ & 0.87 \\
\hline Parahippocampus & $0.4355 \pm 0.0173$ & $0.4177 \pm 0.0315$ & $0.4145 \pm 0.0289$ & 0.71 \\
\hline
\end{tabular}

Mean values \pm standard deviation for all matching variables as well as variables used for correlation analyses. These include sex (where $\mathrm{M}$ stands for male and $\mathrm{F}$ for female); age (in years); education (in terms of educational level on a 0-illiterate-to 5-postsecondary education-scale); Mini-Mental State Examination (MMSE); body mass index (BMI); total physical activity (TPA, normalized by actigraphy wear time); daily average of moderate to vigorous physical activity (MVPA, in minutes); working memory (Forward and Reverse Digit Span — forward and reverse digits); episodic memory (Logical Memory II-delayed recall; for units and gist-logical mem. units and gist); anxiety (Goldberg's test); depression (Geriatric Depression Scale); total gray matter volume (GM, in cubic millimeter); hippocampus, amygdala, and precuneus volumes (left plus right, in cubic millimeter); global fractional anisotropy (FA); and uncinate and parahippocampal fasciculus fractional anisotropy (left and right weighted arithmetic mean). Results are displayed for the whole sample and also for each subsample of interest (APOE $\varepsilon 4$ carriers and non-carriers). $p$ values for the Mann-Whitney and Fisher (sex) tests are also shown. No significant between-group differences arose across all comparisons

the Digit Span Forward, Digit Span Backward, and Logical Memory II (delayed recall, units, and gist) subscales from the Weschler Adult Intelligence Scale IV (WAISIV, $[28])$.

\section{Physical activity measurement}

For PA measurement, we used the ActiGraph GT3X+ accelerometer (LLC, Pensacola, FL). Participants were requested to wear the accelerometers on their right hip for 7 complete days, taking them off only during waterbased activities [29, 30]. For cleaning and processing the data, we used the ActiLife software (6.13.3) (LLC, Pensacola, FL). The validation criteria required each individual to wear the accelerometer during at least 3 weekdays and 1 weekend day for a minimum of $10 \mathrm{~h}$ per day [30]. We considered $\geq 60 \mathrm{~min}$ of continuous zeroes while allowing for up to $2 \mathrm{~min}$ of counts $\leq 100$ counts as nonwear time [31]. To classify the PA, we categorized sedentary time as $<100$ counts/min, light activity as $100-1951$ counts/min, and moderate to vigorous physical activity (MVPA) as $\geq 1952$ counts/min [32].
In this study, two different measures of PA were incorporated: Total Time In Freedson Bouts, which is a standardized measure of PA volumes (total PA, TPA), and daily average of MVPA. TPA was normalized by total wear time.

\section{APOE genotyping}

As described in [23], we obtained genomic DNA from 10 $\mathrm{ml}$ blood samples in ethylenediaminetetraacetic acid (EDTA). Employing TaqMan assays on an Applied Biosystems 7500 Fast Real Time PCR machine (Applied Biosystems, Foster City, CA), single nucleotide polymorphisms (SNPs) rs7412 and rs429358 genotypes were determined. $A P O E$ genotype was established accordingly. In this study, only $\varepsilon 3 \varepsilon 3$ and $\varepsilon 3 \varepsilon 4$ individuals were considered.

\section{MRI acquisition and volumetric analyses}

To generate the T1-weighted MRI images from each participant, a General Electric 1.5-T system was employed. We applied a high-resolution antenna and a homogenization PURE filter (Fast Spoiled Gradient Echo 
sequence, TR/TE/TI $=11.2 / 4.2 / 450 \mathrm{~ms}$; flip angle $12^{\circ} ; 1$ $\mathrm{mm}$ slice thickness, $256 \times 256$ matrix, and FOV $25 \mathrm{~cm}$ ).

The resulting images were processed using the Freesurfer software (version 5.1.0) and its specialized tool for automated cortical parcellation and subcortical segmentation [33]. The measures that were included in further analyses were total gray matter, amygdala, precuneus, and hippocampus (in cubic millimeter). The volumes of bilateral structures were collapsed in order to obtain a single measure for each region.

\section{Diffusion tensor imaging Data acquisition}

The same General Electric 1.5-T magnetic resonance scanner was also used to collect diffusion-weighted images (DWI). The acquisition parameters for DWI were as follows: TE/TR 96.1/12,000 ms; NEX 3 for increasing the SNR; $2.4 \mathrm{~mm}$ slice thickness, $128 \times 128$ matrix, and $30.7 \mathrm{~cm}$ FOV yielding an isotropic voxel of $2.4 \mathrm{~mm}$; 1 image with no diffusion sensitization (i.e., T2-weighted b0 images); and 25 DWI $\left(b=900 \mathrm{~s} / \mathrm{mm}^{2}\right)$. Data were recorded with a single shot echo planar imaging sequence.

\section{Preprocessing}

DWI images were processed following the procedure previously published in [34]. Probabilistic fiber tractography was run on the automated tool AutoPtx (https://fsl. fmrib.ox.ac.uk/fsl/fslwiki/AutoPtx) as reported in [34]. Two bilateral tracts were later used for correlation analyses: the uncinate and the parahippocampal fasciculi. In order to reduce the number of tests, the weighted arithmetic mean of left and right structures was used. Likewise, a measure of global fractional anisotropy (FA) was calculated averaging all 27 original tracts provided by the system.

\section{Magnetoencephalography \\ Data acquisition and signal preprocessing}

MEG data was recorded using a 306-channel wholehead MEG system (Vectorview, ElektaNeuromag, Finland), placed in a magnetically shielded room at the Center for Biomedical Technology in Madrid, following the protocol described in [23].

Raw data were first submitted to the Maxfilter software to remove external noise [35]. Fieldtrip software [36] was used to automatically scan MEG data for artifacts, which were visually confirmed by an MEG expert. Artifact-free data were segmented in $4 \mathrm{~s}$ epochs. Then, MEG time series were filtered into delta $(2-4 \mathrm{~Hz})$, theta $(4-8 \mathrm{~Hz})$, alpha $(8-12 \mathrm{~Hz})$, and beta $(12-30 \mathrm{~Hz})$. This procedure has been reported in detail in [22].

\section{Source reconstruction and connectivity analyses}

We used a regular $1-\mathrm{cm}$ grid in the Montreal Neurological Institute (MNI) template. The resulting model comprised 2459 sources distributed across the brain, which were transformed to each subject's space following the methodology detailed in [22].

We used phase locking value (PLV) to calculate functional connectivity. The Automated Anatomical Labeling atlas (AAL, [37]) was applied to segment the source template with 2459 nodes excluding the cerebellum, basal ganglia, thalamus, and olfactory cortices. The resulting 78 regions of interest included 1202 nodes. Symmetrical whole-brain matrices of $1202 \times 1202$ nodes were obtained by averaging PLV values across trials for each participant and frequency band. Each node's strength was computed by averaging its corresponding FC with the whole grid. Such averaging resulted in a sourcereconstructed FC matrix of 1202 nodes by 4 frequency bands by 107 participants.

\section{Statistical analyses \\ Functional connectivity strength clustering}

Network-based statistics (NBS) were carried out for each frequency band [38]. Clusters consisted of several spatially adjacent nodes that presented a significant partial correlation (age as covariate) between functional connectivity strength (FC-st) values and each PA variable (Spearman's correlation, $p<0.01$ ). To form a cluster, the correlation coefficients of all nodes within the cluster were required to have the same sign. Only clusters including at least $1 \%$ of the grid (i.e., a minimum of 12 nodes) were considered (i.e., minimum size condition). Spearman's rho values were Fisher $Z$-transformed. Cluster-mass statistics were computed as the sum of all $Z$ values corresponding with all nodes within each cluster. Moderation analyses were carried out to study the possible influence exerted by $A P O E$ genotype ( $\varepsilon 4$ carriers vs non-carriers) in the reported relationship between FC-st and TPA. We employed multiple regression analysis and calculated the increase in variance explained by our model after including the interaction term $\left(A P O E_{-}\right.$ by_TPA). This model first used TPA and $A P O E$ as predictors to linearly estimate FC-st in the significant cluster. Then, the interaction term was added (TPA_by_ $A P O E)$. The $p$ value for this interaction term is interpreted as the moderating effect significance.

\section{Controlling for multiple comparisons}

To control for multiple comparisons, the whole process was repeated 5000 times, shuffling the correspondence between FC-st and each PA measure across all participants. At each repetition, the maximum surrogate cluster's statistic was kept creating a maximal null permutation distribution. For each main cluster, cluster- 
mass statistics in the original and the randomized datasets were compared. In NBS, $p$ value represents the proportion of the permutation distribution with clustermass statistic values greater or equal than the clustermass statistic value of the original data. Only clusters which survived NBS (permutation $p$ value $<0.05$ ) were considered in further analyses. For each main cluster, FC-st values were averaged across all nodes to obtain cluster's representative MEG markers.

\section{Correlations between FC-st and markers of brain function and structure}

These markers were used in subsequent correlation analyses with measures of specific AD signatures (the complete list is shown in Table 4). These were carried out taking the whole sample and following stratification of the cohort by $A P O E \varepsilon 4$ carriers and non-carriers. $p$ values were corrected using false discovery rate (FDR) to account for multiple testing. All statistical analyses were carried out using MATLAB R2018b (Mathworks Inc).

\section{Seed-based analyses}

In order to examine whether the FC-st results were caused by global or between-region-specific effects, we performed corresponding seed analysis, using the previous clusters as seeds. The FC values assessed were the average FC between each node of the grid and corresponding cluster's nodes. Then, a set of partial correlation (age as covariate) between these $\mathrm{FC}$ values and each PA variable (Spearman's correlation, $p<0.01$ ) were computed. Only clusters that did not overlap with the original seed cluster were reported in this study.

\section{Results \\ Physical activity is associated with decreased temporal lobe FC-st in $\varepsilon 4$ carriers and non-carriers}

FC-st is computed as the average FC between each specific source and the rest of the network. Here, we examined whether any brain regions, henceforth referred to as clusters, had FC-st values that significantly correlated with PA levels, using age as a covariate. Significant clusters comprised brain regions that behave as functional units.

Applying NBS methodology independently for each frequency band, three significant clusters emerged, located mainly on the left temporal lobe (see Table 2). Using TPA, we found two significant main $(\mathrm{m})$ clusters, one in the theta band (mӨTPA, Fig. 1) and one in the delta band (mסTPA, Fig. 2a). In the case of MVPA, only one cluster in the delta band showed a significant correl-

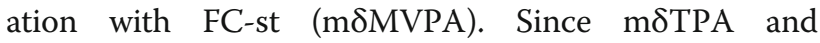

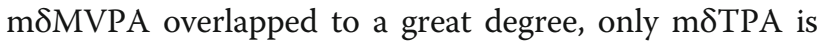
depicted in Fig. 2. In the three clusters, FC-st negatively correlated with PA; thus, higher levels of PA were associated with lower cluster FC-st. In addition, the correlation between PA and FC-st in both delta band clusters remained significant when looking at the $A P O E \varepsilon 4$ carrier and non-carrier groups separately. The cluster in the theta band was significant among $A P O E$ \&4 carriers; however, within the APOE $\varepsilon 4$ non-carriers, the

Table 2 Main clusters presenting decreased FC-st at higher PA levels

\begin{tabular}{|c|c|c|c|}
\hline Cluster & mӨTPA & mঠTPA & mঠMVPA \\
\hline \multirow[t]{13}{*}{ ROls } & Left amygdala (100\%) & Left amygdala (100\%) & Left fusiform gyrus (13.3\%) \\
\hline & Left hippocampus (20\%) & Left fusiform gyrus (13.3\%) & Left inferior frontal gyrus, orbital (33.3\%) \\
\hline & Left inferior frontal gyrus, orbital (8.3\%) & Left inferior frontal gyrus, orbital (41.7\%) & Left inferior temporal gyrus (16.7\%) \\
\hline & Left inferior temporal gyrus (4.2\%) & Left inferior frontal gyrus, opercular (28.6\%) & Left insula (50\%) \\
\hline & Left insula (50\%) & Left inferior temporal gyrus (12.5\%) & Left middle temporal gyrus (13.6\%) \\
\hline & Left middle temporal gyrus (15.9\%) & Left insula (71.4\%) & $\begin{array}{l}\text { Left temporal pole, middle temporal gyrus } \\
(85.7 \%)\end{array}$ \\
\hline & Left postcentral gyrus (2.9\%) & Left middle temporal gyrus (27.3\%) & $\begin{array}{l}\text { Left temporal pole, superior temporal gyrus } \\
(70 \%)\end{array}$ \\
\hline & Left superior temporal gyrus (50\%) & Left parahippocampus (25\%) & Left superior temporal gyrus (15\%) \\
\hline & $\begin{array}{l}\text { Left temporal pole, middle temporal gyrus } \\
(28.6 \%)\end{array}$ & Left postcentral gyrus (11.8\%) & \\
\hline & \multirow{4}{*}{$\begin{array}{l}\text { Left temporal pole, superior temporal gyrus } \\
(40 \%)\end{array}$} & Left rolandic operculum (40\%) & \\
\hline & & Left superior temporal gyrus (45\%) & \\
\hline & & $\begin{array}{l}\text { Left temporal pole, middle temporal gyrus } \\
(85.7 \%)\end{array}$ & \\
\hline & & $\begin{array}{l}\text { Left temporal pole, superior temporal gyrus } \\
(70 \%)\end{array}$ & \\
\hline
\end{tabular}

Total physical activity (TPA) and daily average of moderate to vigorous physical activity (MVPA) correlated with functional connectivity strength (FC-st) in three main clusters. The list of regions of interest (ROIs) upon each significant main cluster fall is shown (in alphabetical order). The percentage of each ROI captured by each cluster is presented in brackets 


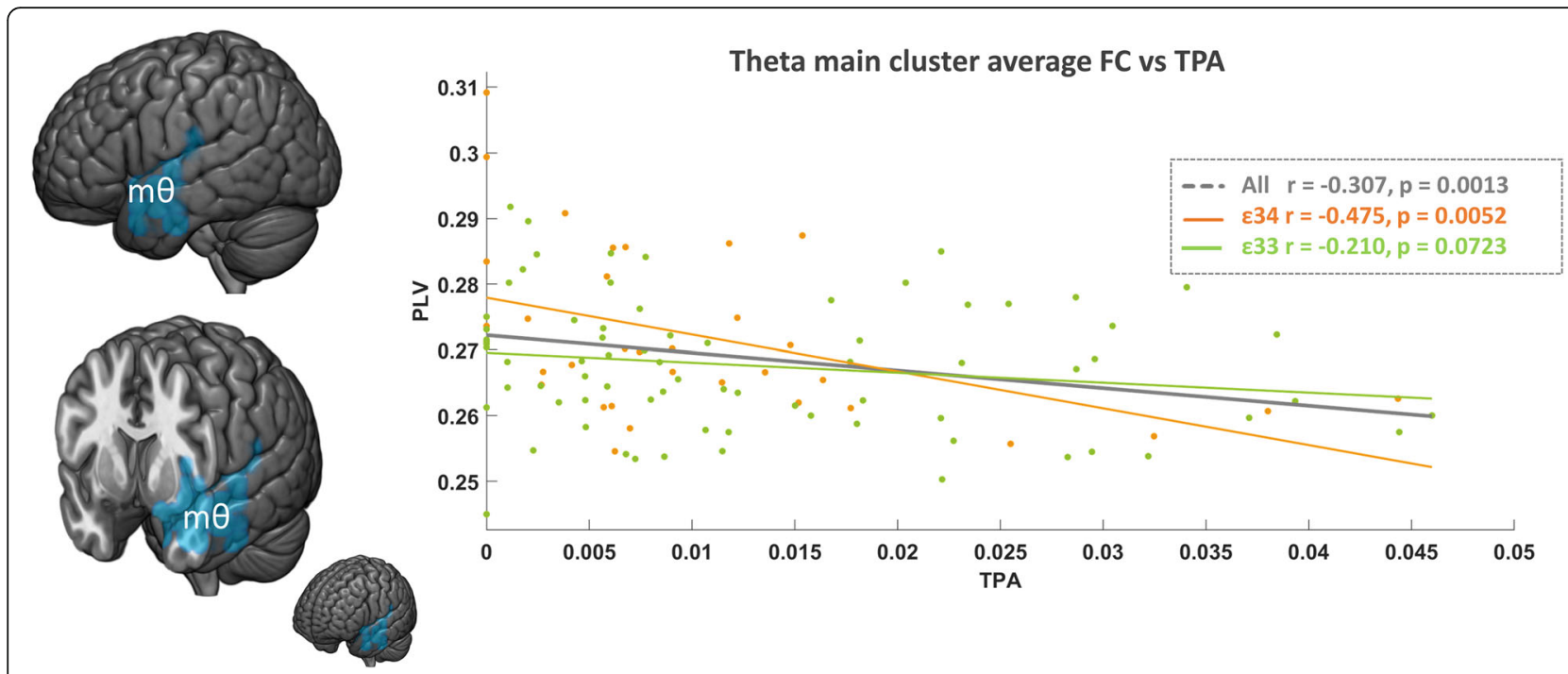

Fig. $1 \mathrm{FC}$-st results in the theta band. In dark blue, marked as $m \theta$, the brain region whose functional connectivity strength (FC-st) was found inversely correlated with total physical activity (TPA) is displayed. On the right, the scatter plot shows the correlation between m $\theta F C$-st and TPA computed with the whole sample (gray), APOE $\varepsilon 4$ carriers (orange), and non-carriers (green)

relationship was not significant. To further assess these potential interaction effects, we conducted a moderation analysis. We observed a significant moderation effect of $A P O E$ genotype for the $\mathrm{m} \theta \mathrm{TPA}$ cluster $(p=0.044)$ while no significant effect was observed for $\operatorname{m} \delta \mathrm{TPA}(p=0.13)$ nor mסMVPA $(p=0.055)$.

Within the current study, greater levels of PA are associated with lower left temporal functional connectivity in both $A P O E \& 4$ carriers and non-carriers. Our findings are relevant to $\mathrm{AD}$ risk, as prodromal $\mathrm{AD}$ is usually characterized, in low frequency bands, as a stage of temporal lobe hyperexcitability [18-22].

\section{Decreased left temporal FC-st is mainly driven by reduced temporo-occipital and temporo-frontal FC}

Decreased cluster FC-st indicates that the oscillatory activity (within a given frequency band) of the cluster regions is less synchronously paired with activity from all across the brain. However, in order to more specifically identify which connections drove such an effect, we performed a seed-based analysis. In this seed-based correlation analysis, we identified the specific connections (secondary clusters) of each of the main clusters with the rest of the brain that were significantly modulated by PA. We found two significant secondary (s) clusters for

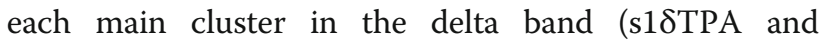
s2 $\delta$ TPA for m $\delta$ TPA, Fig. $2 b$, c, respectively, and

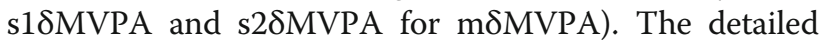
list of areas belonging to these clusters is shown in Table 3. This result was significant among the whole sample and both APOE $\varepsilon 4$ carriage subgroups. No significant secondary clusters emerged for $\mathrm{m} \theta \mathrm{TPA}$.
Lower temporal lobe $\mathrm{FC}$ is differently associated with cognitive function and brain structure in APOE $\varepsilon 4$ carriers and non-carriers

Once we had described how greater levels of PA related to a distinctive FC-st profile, we aimed to characterize the relationship between this profile and parameters of brain health in order to better understand our results. Significant correlations were quite consistent across clusters. In the whole sample, most AD markers negatively correlated with FC-st, so that lower FC-st values were associated with healthier scores over different domains. Additionally, structural measures (total gray matter, hippocampus, precuneus, and amygdala volumes, as well as parahippocampal fasciculus fractional anysotropy) were significantly negatively associated with FC-st among $A P O E \varepsilon 4$ carriers. Anxiety levels also significantly correlated with FC-st in this group, so that lower FC-st was associated with lower anxiety levels. In contrast, only a few significant correlations were found in $A P O E$ $\varepsilon 4$ non-carriers, all of them related to cognition (working and episodic memory). The complete set of correlation results can be found in Table 4 .

\section{Discussion}

The purpose of the current study was to deepen our understanding of the role that $A P O E \varepsilon 4$ plays as a modulator in the relationship between PA and brain structure and function. The most relevant finding of the present work is that greater engagement in PA is related to lower left temporal FC, both in $A P O E$ \&4 carriers and non-carriers. Similar results were obtained with volumes of both total PA and PA at moderate to vigorous intensity. This FC profile was correlated with varying 


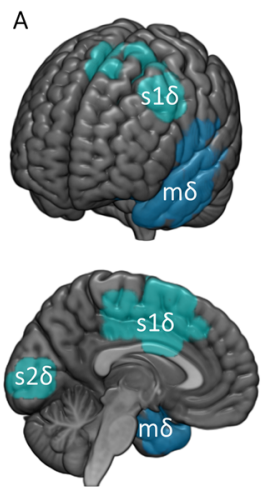

B

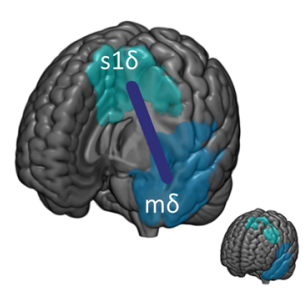

C

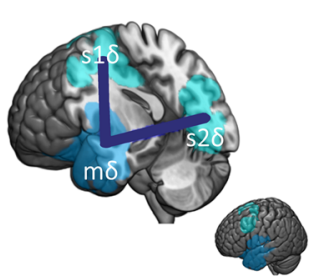

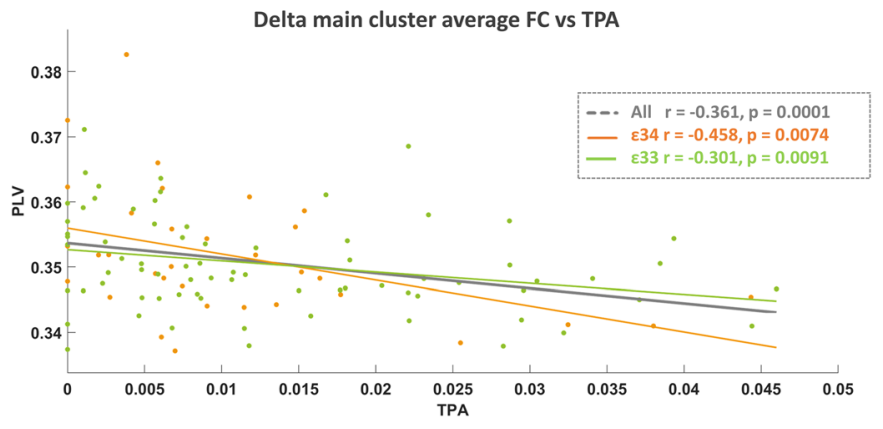

Delta $m \delta$-s $1 \delta$ average FC vs TPA

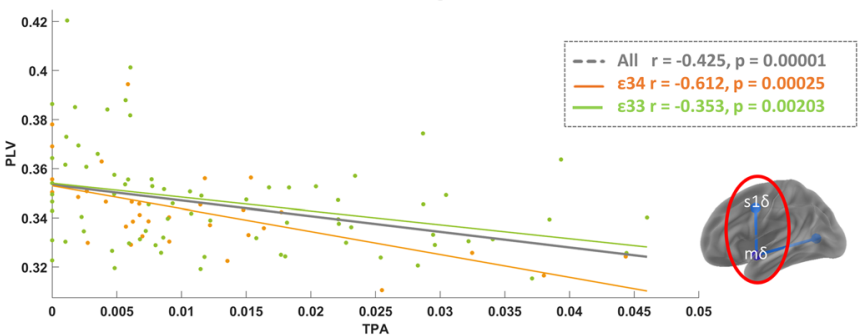

Delta $\mathrm{m} \delta$-s2 $\delta$ average FC vs TPA

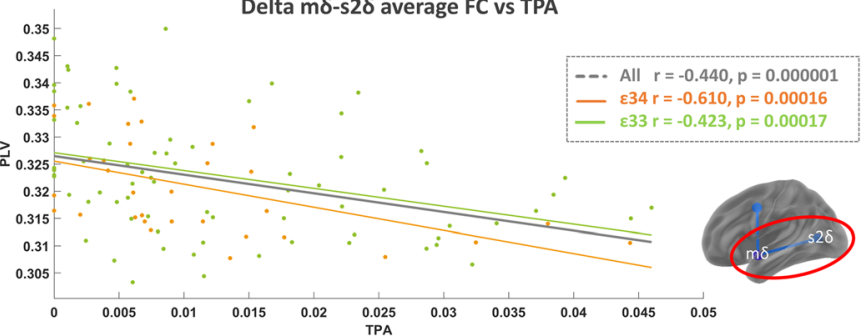

Fig. 2 FC-st results in the delta band. a In dark blue, marked as $m \delta$, the brain region whose functional connectivity strength (FC-st) was found inversely correlated with total physical activity (TPA) is displayed. In light blue, those regions, marked as s $1 \delta$ and s $2 \delta$, whose FC with m $\delta$ was found to inversely correlate with TPA are depicted. On the right, the scatter plot shows the correlation between m $\delta$ FC-st and TPA computed with the whole sample (gray), APOE \&4 carriers (orange), and non-carriers (green). b Seed 1 results (s18). Purple line represents the significant FC link whose value is included in the correlation analysis. The correlation between $\mathrm{m} \delta<->$ s1 $\delta$ FC and TPA is shown on the right. c Seed 2 results (s28). Purple line represents the significant FC link whose value is included in the correlation analysis. The correlation between $\mathrm{m} \delta<->\mathrm{s} 2 \delta \mathrm{FC}$ and physical activity is shown on the right

beneficial effects in AD-related features in both $A P O E$ $\varepsilon 4$ carriers and non-carriers. However, these favorable associations differed according to $\mathrm{AD}$ genetic risk. More specifically, we found a relationship between region-specific decrease in FC-st and greater total GM volumes, greater integrity of the uncinate fasciculus, higher episodic and working memory scores, and reduced anxiety levels across the whole sample. In the $A P O E \& 4$ non-carriers only, network profile correlated with enhanced episodic and working memory, cognitive skills known to be affected early in the course of $\mathrm{AD}$. In contrast, in $A P O E \varepsilon 4$ carriers, left temporal hypoconnectivity was associated with more preserved brain structure, particularly in areas that are more vulnerable to $\mathrm{AD}$ pathology (hippocampus, precuneus, amygdala, and uncinate tract). Figure 3 summarizes these results.

Our finding that PA is associated with reduced temporal lobe hypersynchrony in healthy older adults (even in individuals at greater genetic risk for AD) is noteworthy, considering that $\mathrm{AD}$ has been traditionally described as a disconnection syndrome [39]. However, recent evidence is building on the idea that preclinical AD is characterized by a dual neurophysiological profile. Through MEG, it has been discovered that in subjective memory decline and mild cognitive impairment, a state of hypersynchrony precedes the well-known phase of hypoconnectivity [18-22]. A closer look at these individuals' brain microstructure provides a plausible explanation for these chronological changes. At the very early 
Table 3 Seed-based analyses

\begin{tabular}{|c|c|c|c|c|}
\hline \multirow{2}{*}{$\begin{array}{l}\text { Seed } \\
\text { Cluster }\end{array}$} & \multicolumn{2}{|l|}{ mঠTPA } & \multicolumn{2}{|l|}{ mঠMVPA } \\
\hline & 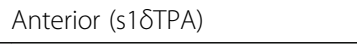 & Posterior (s28TPA) & Anterior (s18MVPA) & Posterior (s28MVPA) \\
\hline \multirow[t]{9}{*}{ ROls } & $\begin{array}{l}\text { Left cingulate gyrus, anterior } \\
\text { part }(10.5 \%)\end{array}$ & $\begin{array}{l}\text { Left calcarine fissure and surrounding } \\
\text { cortex }(20 \%)\end{array}$ & $\begin{array}{l}\text { Left middle frontal gyrus } \\
(11.8 \%)\end{array}$ & $\begin{array}{l}\text { Left calcarine fissure and surrounding } \\
\text { cortex }(20 \%)\end{array}$ \\
\hline & & & Left precentral gyrus (9.4\%) & Left cuneus (9.1\%) \\
\hline & $\begin{array}{l}\text { Left cingulate gyrus, medial } \\
\text { part }(50 \%)\end{array}$ & Left lingual gyrus (7.1\%) & $\begin{array}{l}\text { Left superior frontal gyrus } \\
(18.5 \%)\end{array}$ & Left lingual gyrus (7.1\%) \\
\hline & $\begin{array}{l}\text { Left middle frontal gyrus } \\
(17.6 \%)\end{array}$ & $\begin{array}{l}\text { Right calcarine fissure and } \\
\text { surrounding cortex (50\%) }\end{array}$ & $\begin{array}{l}\text { Left supplementary motor } \\
\text { area }(4.2 \%)\end{array}$ & $\begin{array}{l}\text { Right calcarine fissure and surrounding } \\
\text { cortex }(16.7 \%)\end{array}$ \\
\hline & Left paracentral lobule (33.3\%) & & & Right lingual gyrus (22.2\%) \\
\hline & Left precentral gyrus (15.6\%) & Right cuneus (30.8\%) & & \\
\hline & $\begin{array}{l}\text { Left superior frontal gyrus } \\
(11.1 \%)\end{array}$ & Right lingual gyrus (33.3\%) & & \\
\hline & $\begin{array}{l}\text { Left supplementary motor } \\
\text { area }(50 \%)\end{array}$ & Right middle occipital lobe (17.6\%) & & \\
\hline & $\begin{array}{l}\text { Left superior frontal gyrus, } \\
\text { medial }(5.9 \%)\end{array}$ & Right middle temporal gyrus (5.4\%) & & \\
\hline
\end{tabular}

Each main cluster ( $\mathrm{m} \theta T P A, m \delta T P A$, and $\mathrm{m} \delta M V P A)$ whose functional connectivity strength (FC-st) was significantly correlated with PA was used as a seed in a

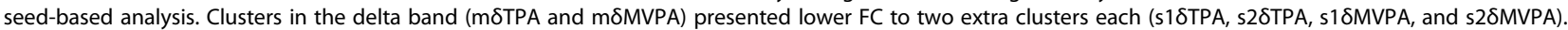
The regions of interest (ROIs) that are comprised in each additional cluster are presented. The percentage of each ROI captured by each cluster is presented in brackets

silent stages of $\mathrm{AD}$ pathology, inhibitory neurons are lost, mainly in middle temporal regions [13, 14]. Such loss of inhibitory synapses leads to a state of brain hyperexcitability and hypersynchrony, which can be tracked through MEG [15]. Sustained hyperactivation elicits neurotoxic effects and increased release of amyloid (which ultimately lead to neuronal damage [16, 17]). As a result, extensive brain atrophy and generalized hypoconnectivity are evident features by the time AD clinical symptomatology arises [40].

It is interesting that it was the left temporal lobe which exhibited significant results, since decreased synchronization in $\mathrm{AD}$, already at the dementia stage, seems to mainly affect the left hemisphere [41]. On the other hand, both normal aging and $\mathrm{AD}$ brain activity presents a marked "slowness," this is an increase in power in low frequency bands (delta and theta) [42]. In fact, increases in delta connectivity had already been described as a pathological sign in other clinical conditions, as well as decreases upon cognitive recovery [43]. It is possible then that since low frequency rhythms are more associated with brain neurophysiological health, PA could exert its beneficial effect by affecting these rhythms specifically. Conversely, although there are not many functional connectivity studies that could provide an explanation on why physical activity affects those frequency bands in particular, power spectrum studies suggest that during an acute bout of exercise, activity in the theta band is enhanced. Such increase in theta power is believed to serve a cognitive function, as physical activity is evolutionary associated with increases in cognitive demands [44]. This effect is usually reversed immediately after the physical activity bout ceases $[45,46]$. This phenomenon could be related to the diminished FC-st within the theta band that we find in active individuals at rest.

In an attempt to understand the meaning of our FC results, we also studied the association between the observed brain activity patterns and cognitive/emotional functioning, brain volumes, and white matter integrity: within these analyses, differences between $A P O E$ \&4 carriers and non-carriers are evident. Previous literature offers mixed results with regard to whether $A P O E \& 4$ carriers or non-carriers gain the greatest benefit from PA engagement. This inconsistent literature may be a result of the utilization of varying PA measurements (questionnaires, fitness measures, and PA interventions) and different outcomes (AD risk, cognitive scores, or brain activity/structure), conducted in samples of different characteristics (in terms of age, AD risk, and cognitive status). In the current study, we examined the effect of objectively measured $\mathrm{PA}$ on a wide range of $\mathrm{AD}$ markers employing a sample of cognitively healthy $A P O E \& 4$ carriers and non-carriers properly matched on an extensive list of potential confounders.

While measures of different brain volumes correlated with $\mathrm{FC}$-st in the $\varepsilon 4$ carrier group, these associations did not exist in $\varepsilon 4$ non-carriers. The same pattern arose when looking at the integrity of the uncinate tract. It is important to highlight that these are all brain structures that are particularly vulnerable to AD pathology. Since this is a sample of cognitively healthy participants, our results could be better understood if we consider that individuals at increased genetic risk already present greater variability in GM and WM state of preservation. Therefore, we could assume that there is more room for PA to 
Table 4 Correlation analyses

\begin{tabular}{|c|c|c|c|c|c|c|c|c|c|c|c|c|c|c|c|c|c|c|}
\hline & \multicolumn{6}{|c|}{ Whole sample } & \multicolumn{6}{|l|}{ Carriers } & \multicolumn{6}{|c|}{ Non-carriers } \\
\hline & \multicolumn{2}{|l|}{ mӨTPA } & \multicolumn{2}{|l|}{ mঠTPA } & \multicolumn{2}{|c|}{ mסMVPA } & \multicolumn{2}{|l|}{ mӨTPA } & \multicolumn{2}{|l|}{ mঠTPA } & \multicolumn{2}{|c|}{ mסMVPA } & \multicolumn{2}{|l|}{ mӨTPA } & \multicolumn{2}{|l|}{ mঠTPA } & \multicolumn{2}{|c|}{ mSMVPA } \\
\hline & Rho & $p$ & Rho & $p$ & Rho & $p$ & Rho & $p$ & Rho & $p$ & Rho & $p$ & Rho & $p$ & Rho & $p$ & Rho & $p$ \\
\hline Forward digits & -0.20 & 0.03 & -0.18 & 0.061 & -0.20 & 0.04 & -0.30 & 0.09 & -0.08 & 0.66 & -0.04 & 0.84 & -0.17 & 0.14 & -0.23 & 0.05 & -0.28 & 0.01 \\
\hline Reverse digits & -0.23 & 0.02 & -0.21 & 0.03 & -0.22 & 0.03 & -0.29 & 0.11 & -0.23 & 0.20 & -0.22 & 0.23 & -0.23 & 0.05 & -0.22 & 0.06 & -0.22 & 0.06 \\
\hline Logical mem. units & -0.00 & 0.96 & -0.00 & 0.99 & -0.03 & 0.78 & -0.11 & 0.54 & -0.16 & 0.40 & -0.12 & 0.51 & 0.05 & 0.65 & 0.09 & 0.47 & 0.06 & 0.63 \\
\hline $\begin{array}{l}\text { Logical } \\
\text { mem. gist }\end{array}$ & -0.18 & 0.07 & -0.27 & 0.01 & -0.23 & 0.02 & -0.09 & 0.62 & -0.13 & 0.51 & -0.09 & 0.66 & -0.20 & 0.10 & -0.34 & 0.01 & -0.30 & 0.01 \\
\hline Anxiety & 0.29 & $<0.01$ & 0.22 & 0.03 & 0.2 & 0.05 & 0.46 & 0.01 & 0.28 & 0.15 & 0.29 & 0.13 & 0.20 & 0.09 & 0.19 & 0.13 & 0.16 & 0.18 \\
\hline Depression & 0.08 & 0.423 & 0.08 & 0.43 & 0.079 & 0.44 & -0.04 & 0.84 & -0.06 & 0.72 & -0.11 & 0.54 & 0.14 & 0.25 & 0.17 & 0.17 & 0.20 & 0.10 \\
\hline Total GM & -0.27 & 0.01 & -0.29 & $<0.01$ & -0.29 & $<0.01$ & -0.39 & 0.03 & -0.44 & 0.01 & -0.43 & 0.02 & -0.23 & 0.05 & -0.23 & 0.05 & -0.23 & 0.05 \\
\hline Amygdala & -0.28 & $<0.01$ & -0.27 & $<0.01$ & -0.27 & $<0.01$ & -0.33 & 0.07 & -0.41 & 0.02 & -0.39 & 0.03 & -0.25 & 0.03 & -0.21 & 0.08 & -0.21 & 0.07 \\
\hline Hippocampus & -0.26 & 0.01 & -0.27 & $<0.01$ & -0.28 & $<0.01$ & -0.50 & $<0.01$ & -0.47 & 0.01 & -0.42 & 0.02 & -0.16 & 0.18 & -0.17 & 0.15 & -0.20 & 0.08 \\
\hline Precuneus & -0.14 & 0.15 & -0.20 & 0.04 & -0.21 & 0.03 & -0.15 & 0.41 & -0.42 & 0.02 & -0.42 & 0.02 & -0.11 & 0.35 & -0.10 & 0.42 & -0.10 & 0.41 \\
\hline Global FA & -0.03 & 0.72 & -0.08 & 0.43 & -0.04 & 0.69 & -0.18 & 0.33 & -0.16 & 0.38 & -0.11 & 0.56 & -0.00 & 0.97 & -0.04 & 0.73 & -0.00 & 0.98 \\
\hline Uncinate & -0.24 & 0.02 & -0.18 & 0.07 & -0.14 & 0.16 & -0.61 & $<0.01$ & -0.44 & 0.01 & -0.39 & 0.03 & -0.07 & 0.58 & -0.07 & 0.59 & -0.01 & 0.91 \\
\hline Parahippocampus & -0.00 & 0.96 & -0.07 & 0.49 & -0.03 & 0.76 & -0.17 & 0.37 & -0.30 & 0.10 & -0.22 & 0.23 & 0.05 & 0.68 & 0.03 & 0.77 & 0.07 & 0.55 \\
\hline
\end{tabular}

counterbalance early neuropathological signs within this group. This is consistent with previous studies that demonstrate the effect of PA on brain pathology is most predominant among $\varepsilon 4$ carriers [7, 47, 48].

Contrary to our aforementioned findings within $A P O E$ $\varepsilon 4$ carriers, we observed a relationship between decreased FC and greater scores in specific measures of episodic and working memory, but only among APOE \&4. non-carriers. Most studies investigating the relationship between $\mathrm{AD}$ incidence and PA have concluded that only $A P O E$ \&4 non-carriers benefit from reduced AD risk at greater levels of PA $[9,49,50]$, although there are some exceptions [51, 52]. Presently, AD diagnosis is based on clinical progression and cognitive status. Therefore, our finding that the profile of FC associated with PA only predicts cognitive functioning among $A P O E \& 4$ noncarriers is somewhat consistent with previous research.

Finally, across the whole sample and APOE \&4 carriers, we found a positive correlation between greater FC-st and higher anxiety. In previous studies, anxiety has been identified as a marker of conversion from preclinical AD to $\mathrm{AD}[53,54]$. In addition, higher levels of anxiety are related to greater temporal lobe atrophy [55]. Hence, our results demonstrate that PA-associated reduced FCst is also associated with lower anxiety levels and greater temporal lobe volumes.

PA has been widely studied as a protective ally against AD. Our study sheds light on the potential mechanisms through which PA could exert its action. According to the neurogenic reserve hypothesis, throughout evolution acute bouts of PA were linked to an increased likelihood of a potential cognitive challenge [56, 57]. As huntergatherers, going through long distances relied on improved spatial orientation, memory, and executive functions. Locomotion would signal the brain such increase in cognitive demands. In response, the reserve of neuronal precursor cells would grow. In the presence of cognitive stimulation, new neurons would maturate, differentiate, and migrate. Multiple sources of evidence support the postulate that PA promotes synaptogenesis and neurogenesis, mainly within the hippocampal network $[58,59]$. Newborn granule cells do not produce hyperactivation but rather present sparse activity during learning [60]. Such mechanisms could explain the decreased temporal FC profile that we detect in older adults who regularly engage in greater levels of PA. But most importantly, they could explain why PA is one the most relevant modifiable protective factor against $\mathrm{AD}$. Indeed, Raicheln and Alexander hypothesize that 2 million years ago, PA was able to counterbalance the detrimental effects of $A P O E \& 4$, when our ancestors carried two copies of this risk allele [61]. However, although in this study we report that the association between PA and FC-st is associated to beneficial effects in both $\varepsilon 4$ carriers and non-carriers, it remains possible that the underlying mechanisms differ based on AD genetic risk.

The results observed in the current study provide a more comprehensive picture of the relationship between 


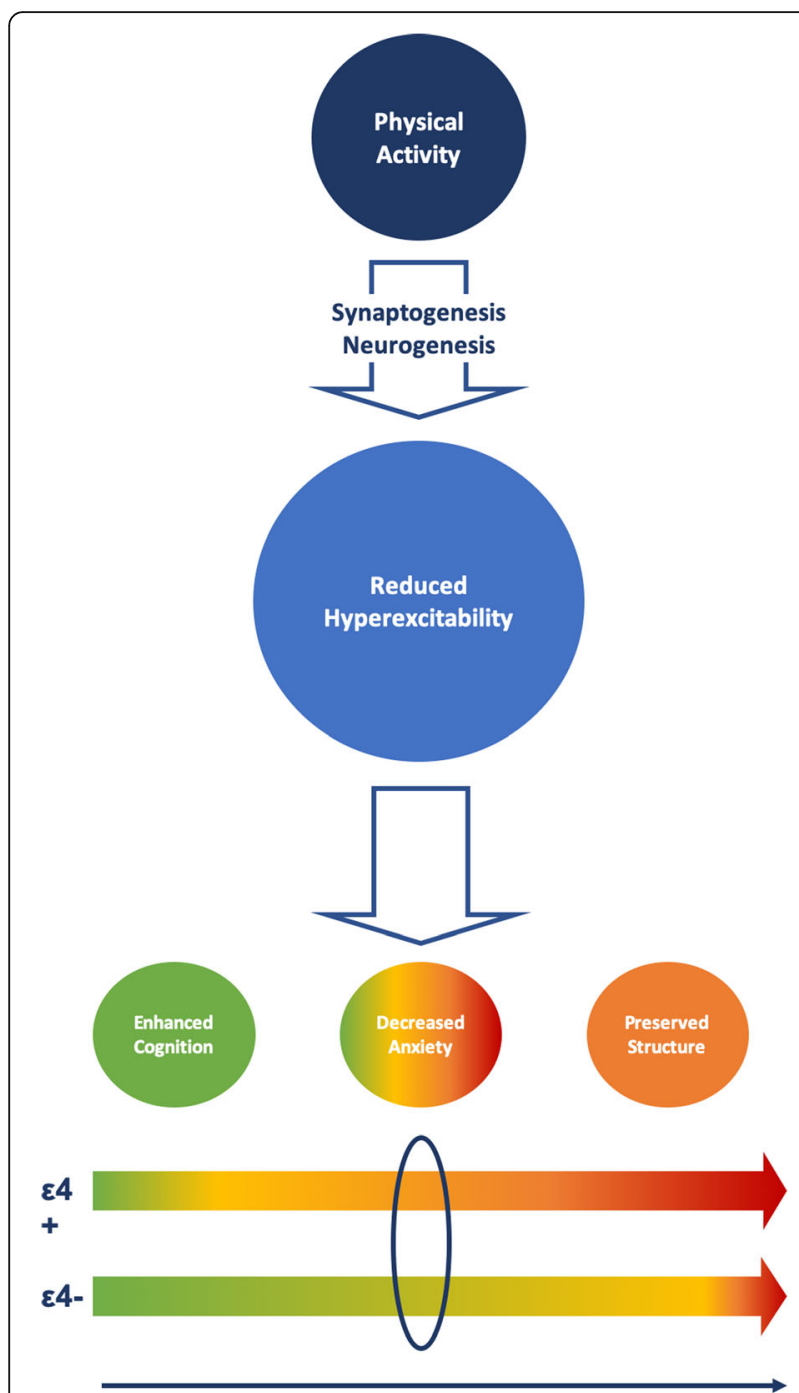

Aging Process

Fig. 3 Proposed mechanism for physical activity-induced beneficial effects on brain health in APOE $\varepsilon 4$ carriers and non-carriers. Physical activity (PA) is associated with decreased functional connectivity (FC) both in APOE $\varepsilon 4$ carriers and non-carriers. We propose that this relationship could be mediated by a PA-induced increase in neurogenesis and synaptogenesis. Such processes could in turn prevent the loss of inhibitory synapses that has been identified to cause hyperexcitability in temporal regions in prodromal Alzheimer's disease (AD). Interestingly, this decrease in FC manifests differently according to AD genetic risk. In $\varepsilon 4$ carriers, this profile is linked to reduced anxiety and preserved brain structure. Conversely, in $\varepsilon 4$ non-carriers, it is associated with enhanced cognition. One possibility behind this pattern of results could be that $\varepsilon 4$ carriers were at higher risk of neuronal damage, which in normal aging would appear later. Therefore, at the specific time when we are taking these measurements, PA has more room to exert its beneficial effect on brain structure in $\varepsilon 4$ carriers, while in non-carriers, at lower risk for neuropathological burden, it is associated with improved cognitive functioning. Hence, it remains plausible that at older ages, PA could also relate to greater structural integrity. However, we cannot rule out the possibility that PA affected $\varepsilon 4$ carriers and non-carriers through different mechanisms
PA, APOE, and AD pathology, compared with previously conducted studies. Our findings help strengthen the understanding of the complex dynamics that underpin the varying outcomes observed in previous studies. Although the age range in this sample was fairly broad, the mean age was still quite low to study the effects of advanced aging or prodromal AD. Therefore, it is possible that we missed out on certain effects that might only appear at later stages of life. Follow-up studies are required to determine how the FC profiles identified within this study are associated with pathological progression and cognitive change among, at-present, cognitively healthy older adults. Also, future studies should include a group of $A P O E \varepsilon 4$ homozygotes, since our sample size did not allow us to incorporate that comparison. Additionally, it would be interesting to see how diverse PA parameters, such as the type of activity or frequency of practice, affect certain markers of the disease. This information could be useful in the elaboration of lifestyle guidelines aiming to promote brain health. Unfortunately, such measures were not available from this cohort. In addition, it would be useful to include specific AD biomarkers to better characterize brain health in individuals at risk, instead of relying solely in genetic risk factors to identify individuals at greater risk of developing dementia.

\section{Conclusions}

Altogether, our study offers novel insights into this field, clarifying some of the specific mechanisms that underlie the beneficial effect of PA in $A P O E \varepsilon 4$ carriers and noncarriers. It enables the integration of previous publications and leads the way to future findings. Previous literature offered apparently inconsistent results, but our study suggests that the specific brain health outcomes considered could be differently affected by PA in $\varepsilon 4$ carriers and non-carriers. Nevertheless, we were able to describe an association between PA and decreased temporal lobe hypersynchrony across the whole sample that highlights the need to design new policies that foster PA among older adults, including those more vulnerable to develop AD.

\section{Abbreviations}

AAL: Automated Anatomical Labeling atlas; AD: Alzheimer's disease; APOE: Apolipoprotein E; DWI: Diffusion-weighted images;

EDTA: Ethylenediaminetetraacetic acid; FA: Fractional anisotropy; FC: Functional connectivity; FC-st: Functional connectivity strength; FDR: False discovery rate; GM: Gray matter; mӨTPA: Main cluster found in the theta band using TPA; mSTPA: Main cluster found in the delta band using TPA; mסMVPA: Main cluster found in the delta band using MVPA; MEG: Magnetoencephalography; MNI: Montreal Neurological Institute; MVPA: Moderate to vigorous physical activity; NBS: Network-based statistics; PA: Physical activity; PLV: Phase locking value; ROI: Region of interest; TPA: Total physical activity; s18TPA and s2 $2 T P A:$ Secondary clusters

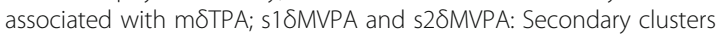
associated with mSMVPA; SNP: Single nucleotide polymorphism; WAISIV: Weschler Adult Intelligence Scale IV 


\section{Acknowledgements}

Not applicable.

\section{Authors' contributions}

$F M, R L H$, and AM outlined the research project. JFL, PC, and DLS designed the experiments. FRT, ICRR, and JFL coordinated the data collection. FRT and JFL processed the MEG recordings. RB developed the MEG processing pipeline. AMLS, APS, and ECS contributed the actigraphy data. MLDL coordinated the neuropsychological assessment. AB performed the genotyping analyses. JVR provided the DTI analysis methodology. PC performed the statistical analyses and prepared the figures. JFL drafted the original manuscript, and PC, DLS, BB, JMS, SML, and FM revised the original draft and contributed relevant suggestions to the final manuscript. All authors revised and approved the final paper draft.

\section{Funding}

This study was funded by the Spanish Ministry of Economy and Competitiveness under the Grant PSI2015-68793-C3-1-R [D601] and by the project B2017/BMD-3760 from NEUROCENTRO. Complimentary, it was supported by a predoctoral fellowship from La Caixa Foundation to JFL, a postdoctoral fellowship from the Spanish Ministry of Economy and Competitiveness to PC (FJCl-2015-26755), a grant from the Spanish Ministry of Science, Innovation and Universities to JVR (FJCl-2017-33396), and a predoctoral grant by the Spanish Ministry of Economy (BES-2016-076869) to FRT.

\section{Availability of data and materials}

The datasets used and/or analyzed during the current study are available from the corresponding author on reasonable request.

\section{Ethics approval and consent to participate}

The procedure was performed following current guidelines and regulations, and the study was approved by the Hospital Universitario San Carlos Ethics Committee under the code 15/382-E_BS. Every participant signed an informed consent.

\section{Consent for publication}

Not applicable.

\section{Competing interests}

None of the contributors to this study have any conflict of interest to declare.

\section{Author details}

'Biological and Health Psychology Department, School of Psychology, Universidad Autonoma de Madrid, 28049 Madrid, Spain. ${ }^{2}$ Laboratory of Cognitive and Computational Neuroscience (UCM-UPM), Center for biomedical Technology, Parque Científico y Tecnológico de la UPM, Crta. M40, Km. 38, 28223 Pozuelo de Alarcón, Madrid, Spain. ${ }^{3}$ Collaborative Genomics Group, School of Medical and Health Sciences, Edith Cowan University, Joondalup, Western Australia 6027, Australia. ${ }^{4}$ Department of Industrial Engineering \& IUNE, Universidad de La Laguna, 38200 San Cristobal de la Laguna, Tenerife, Spain. ${ }^{5}$ Department of Psychobiology and Methodology in Behavioral Sciences, School of Education, Universidad Complutense de Madrid, 28040 Madrid, Spain. ${ }^{6}$ Departamento de Nutricion y Ciencia de los Alimentos, Facultad de Farmacia, Universidad Complutense de Madrid, 28040 Madrid, Spain. ${ }^{7}$ Experimental Psychology Department, School of Psychology, Universidad Complutense de Madrid, 28223 Pozuelo de Alarcon, Spain. ${ }^{8}$ Discipline of Exercise Science, College of Science, Health, Engineering and Education, Murdoch University, Murdoch, Western Australia 6150, Australia. ${ }^{9}$ School of Pharmacy and Biomedical Sciences, Faculty of Health Sciences, Curtin Health Innovation Research Institute, Curtin University, Bentley, Western Australia 6102, Australia. ${ }^{10}$ Centro Universitario Villanueva, Facultad de Psicología, 28034 Madrid, Spain. ${ }^{11}$ Mind, Brain and Behavior Research Center (CIMCYC), Universidad de Granada, 18071 Granada, Spain. ${ }^{12}$ Networking Research Center on Bioengineering, Biomaterials and Nanomedicine (CIBER-BBN), 28029 Madrid, Spain. ${ }^{13}$ Endocrinology and Nutrition Department, Hospital Clinico San Carlos and Instituto de Investigación Sanitaria del Hospital Clínico San Carlos, 28040 Madrid, Spain. ${ }^{14}$ Centro de Investigación Biomédica en Red de Diabetes y Enfermedades Metabólicas Asociadas, 28040 Madrid, Spain. ${ }^{15}$ Neurology Department,
Hospital Clinico San Carlos and Instituto de Investigación Sanitaria del Hospital Clínico San Carlos, 28040 Madrid, Spain.

Received: 2 February 2020 Accepted: 30 March 2020

Published online: 24 April 2020

\section{References}

1. Piercy KL, Troiano RP, Ballard RM, Carlson SA, Fulton JE, GD A, et al. The physical activity guidelines for Americans. JAMA. 2018;320(19):2020-8.

2. Erickson Kl, Weinstein AM, Lopez OL. Physical activity, brain plasticity, and Alzheimer's disease. Arch Med Res. 2012;43(8):615-21 Available from: https://doi.org/10.1016/j.arcmed.2012.09.008.

3. Okonkwo OC, Schultz SA, Oh JM, Larson J, Edwards D, Cook D, et al. Physical activity attenuates age-related biomarker alterations in preclinical AD. Neurology. 2014;83(1753):1760.

4. Brown BM, Rainey-Smith SR, Dore V, Pfeiffer JJ, Burnham SC, Laws SM, et al. Self-reported physical activity is associated with tau burden measured by positron emission tomography. J Alzheimers Dis. 2018;63(4):1299-305.

5. Farrer LA, Cupples LA, Haines JL, Hyman B, Kukull WA, Mayeux R, et al. Effects of age, sex, and ethnicity on the association between apolipoprotein E genotype and Alzheimer disease. JAMA. 1997;278(16):22-9.

6. Thibeau S, Mcfall GP, Camicioli R, Dixon RA. Alzheimer's disease biomarkers interactively influence physical activity, mobility, and cognition associations in a non-demented aging population. J Alzheimers Dis. 2017;60:69-86.

7. Cerin E, Rainey-smith SR, Ames D, Lautenschlager NT, Macaulay SL, Fowler $C$, et al. Associations of neighborhood environment with brain imaging outcomes in the Australian Imaging, Biomarkers and Lifestyle cohort. Alzheimers Dement. 2017;13(4):388-98 Available from: https://doi.org/10. 1016/j.jalz.2016.06.2364.

8. Podewils LJ, Guallar E, Kuller LH, Fried LP, Lopez OL, Carlson M, et al. Physical activity, APOE genotype, and dementia risk: findings from the cardiovascular health cognition study. Am J Epidemiol. 2005;161(7):639-51.

9. Tan ZS, Spartano NL, Beiser AS, Decarli C, Auerbach SH, Vasan RS, et al. UCLA physical activity, brain volume, and dementia risk: the Framingham study. J Gerontol Ser A Biomed Sci Med Sci. 2016;72(6):789-95.

10. Lindsay J, Laurin D, Verreault R, Hébert R, Helliwell B, Hill GB, et al. Risk factors for Alzheimer's disease: a prospective analysis from the Canadian study of health and aging. Am J Epidemiol. 2002;156(5):445-53.

11. Stern Y, Mackay-Brandt A, Lee S, Mckinley P, Mclntyre K, Razlighi Q, et al. Effect of aerobic exercise on cognition in younger adults: a randomized clinical trial. Neurology. 2019;92(9):905-16.

12. Bastos AM, Schoffelen J-M. A tutorial review of functional connectivity analysis methods and their interpretational pitfalls. Front Syst Neurosci. 2016;9(January):1-23.

13. Varela EV, Etter G, Williams S. Excitatory-inhibitory imbalance in Alzheimer's disease and therapeutic significance. Neurobiol Dis. 2019;127(July):605-15 Available from: https://doi.org/10.1016/j.nbd.2019.04.010.

14. Najm R, Jones EA, Huang Y. Apolipoprotein E4, inhibitory network dysfunction, and Alzheimer's disease. Mol Neurodegener. 2019;6(14):24.

15. López-sanz D, Bruña R, de Frutos-Lucas J, Maestú F. Magnetoencephalography applied to the study of Alzheimer's disease. In: Progress in molecular biology and translational science. 1st ed. Amsterdam: Elsevier Inc; 2019. p. 1-37. Available from: https://doi.org/10.1016/bs.pmbts.2019.04.007.

16. Busche MA, Konnerth A. Impairments of neural circuit function in Alzheimer's disease. Philos Trans R Soc Lond Ser B Biol Sci. 2016;371(1700): 20150429.

17. De Haan W, Van Straaten ECW, Gouw AA, Stam CJ. Altering neuronal excitability to preserve network connectivity in a computational model of Alzheimer's disease. PLoS Comput Biol. 2017;13(9):1-23.

18. Lopez ME, Bruna R, Aurtenetxe S, Pineda-Pardo JA, Marcos A, Arrazola J, et al. Alpha-band hypersynchronization in progressive mild cognitive impairment: a magnetoencephalography study. J Neurosci. 2014;34(44): 14551-9.

19. López-Sanz D, Garcés P, Álvarez B, Delgado-Losada ML, López-Higes R, Maestú F. Network disruption in the preclinical stages of Alzheimer's disease: from subjective cognitive decline to mild cognitive impairment. Int Jeural Syst. 2017:27(8):S0129065717500411.

20. Jones D, Knopman DS, Gunter JL, Graff-Radford J, Vemuri P, Boeve BF, et al. Cascading network failure across the Alzheimer's disease spectrum. Brain. 2015;139(2):547-62. 
21. Nakamura A, Cuesta P, Kato T, Arahata Y, Iwata K, Yamagishi M, et al. Early functional network alterations in asymptomatic elders at risk for Alzheimer's disease. Sci Rep. 2017;7(1):1-11.

22. Pusil S, Lopez ME, Cuesta P, Bruña R, Pereda E, Maestu F. Hypersynchronization in mild cognitive impairment: the ' $X$ ' model. Brain. 2019;142(12):1-15.

23. de Frutos-Lucas JD, López-Sanz D, Zuluaga P, Rodríguez-Rojo IC, Luna R, María Eugenia L, et al. Clinical neurophysiology physical activity effects on the individual alpha peak frequency of older adults with and without genetic risk factors for Alzheimer's disease : a MEG study. Clin Neurophysiol. 2018;129(9):1981-9 Available from: https://doi.org/10.1016/j.clinph.2018.06.026.

24. Yamazaki Y, Zhao N, Caulfield TR, Liu CC, Bu G. Apolipoprotein E and Alzheimer disease: pathobiology and targeting strategies. Nat Rev Neurol. 2019;15(9):501-18 Available from: https://doi.org/10.1038/s41582-019-0228-7.

25. Flowers SA, Rebeck GW. APOE in the normal brain. Neurobiol Dis. 2020; 136(January) Available from: https://doi.org/10.1016/j.nbd.2019.104724.

26. Yesavage JA, Brink $T L$, Rose $T L$, Lum $O$, Huang $V$, Adey $M$, et al. Development and validation of a geriatric depression screening scale: a preliminary report. J Psychiatr Res. 1982;17(1):37-49.

27. Herrero MJ, Blanch J, Peri JM, de Pablo J, Pintor L, Bulbena A. A validation study of the hospital anxiety and depression scale (HADS) in a Spanish population. Gen Hosp Psychiatry. 2003;25(4):277-83.

28. Weschler D. Wechsler adult intelligence scale-fourth edition (WAIS-IV). 4th ed. San Antonio: Pearson; 2008.

29. Chudyk AM, McAllister MM, Cheung HK, McKay HA, Ashe MC. Are we missing the sitting? Agreement between accelerometer non-wear time validation methods used with older adults' data. Cogent Med. 2017;4(1):117 Schumacher $U$, editor.

30. Chomistek AK, Yuan C, Matthews CE, Troiano RP, Bowles HR, Rood J, et al. Physical activity assessment with the ActiGraph GT3X and doubly labeled water. Med Sci Sports Exerc. 2017;49(9):1935-44

31. Troiano RP, Berrigan D, Dodd KW, Mâsse LC, Tilert T, Mcdowell M. Physical activity in the United States measured by accelerometer. Med Sci Sports Exerc. 2008;40(1):181-8.

32. Van Dyck D, Herman K, Poppe L, Crombez G, De Bourdeaudhuij I, Gheysen F. Results of MyPlan 2.0 on physical activity in older Belgian adults: randomized controlled trial. J Med Internet Res. 2019;21(10):e13219.

33. Fischl B, Salat DH, Busa E, Albert M, Dieterich M, Haselgrove $C$, et al. Whole brain segmentation: neurotechnique automated labeling of neuroanatomical structures in the human brain. Neuron. 2002;33:341-55.

34. Verdejo-Román J, Björnholm L, Muetzel RL, Torres-Espínola FJ, Lieslehto J, Jaddoe $V$, et al. Maternal prepregnancy body mass index and offspring white matter microstructure: results from three birth cohorts. Int J Obes. 2018; Available from: https://doi.org/10.1038/s41366-018-0268-x.

35. Taulu S, Simola J. Spatiotemporal signal space separation method for rejecting nearby interference in MEG measurements. Phys Med Biol. 2006;51(7):1759-68.

36. Oostenveld R, Fries P, Maris E, Schoffelen J-M. FieldTrip: open source software for advanced analysis of MEG, EEG, and invasive electrophysiological data. Comput Intell Neurosci. 2011;2011:156869.

37. Tzourio-Mazoyer N, Landeau B, Papathanassiou D, Crivello F, Etard O, Delcroix $\mathrm{N}$, et al. Automated anatomical labeling of activations in SPM using a macroscopic anatomical parcellation of the MNI MRI single-subject brain. Neuroimage. 2002;15(1):273-89.

38. Zalesky A, Fornito A, Bullmore ET. Network-based statistic: identifying differences in brain networks. Neuroimage. 2010:53(4):1197-207.

39. Delbeuck X, Van der Linden M, Collette F. Alzheimer' disease as a disconnection syndrome? Neuropsychol Rev. 2003;13(2):79-92.

40. Koelewijn L, Bompas A, Tales A, Brookes MJ, Muthukumaraswamy SD, Bayer A, et al. Alzheimer's disease disrupts alpha and beta-band resting-state oscillatory network connectivity. Clin Neurophysiol. 2017;128(11):2347-57 Available from: https://doi.org/10.1016/j.clinph.2017.04.018.

41. Engels MMA, Yu M, Stam CJ, Gouw AA, van der Flier WM, Scheltens P, et al. Directional information flow in patients with Alzheimer's disease. A sourcespace resting-state MEG study. Neurolmage Clin. 2017;15:673-81.

42. Susi G, De Frutos-Lucas J, Niso G, Ye-chen SM, Toro LA, Vilca Chino NB, et al. Healthy and pathological neurocognitive aging : spectral and functional connectivity analyses using mag netoencephalography. In: Oxford Research Encyclopedia of Psychology; 2019. p. 1-46.

43. Castellanos N, Paúl N, Ordoñez VE, Demuynck O, Bajo R, Campo P, et al. Reorganization of functional connectivity as a correlate of cognitive recovery in acquired brain injury. Brain. 2010;133:2365-81.
44. Horne J. Exercise benefits for the aging brain depend on the accompanying cognitive load: insights from sleep electroencephalogram. Sleep Med. 2013; 14(11):1208-13 Available from: https://doi.org/10.1016/j.sleep.2013.05.019.

45. Kuo TBJ, Li JY, Shen-Yu Hsieh S, Chen JJ, Tsai CY, Yang CCH. Effect of aging on treadmill exercise induced theta power in the rat. Age (Omaha). 2010; 32(3):297-308

46. Li JY, Kuo TBJ, Hsieh SSY, Yang CCH. Changes in electroencephalogram and heart rate during treadmill exercise in the rat. Neurosci Lett. 2008;434(2):175-8.

47. Smith JC, Nielson KA, Woodard JL, Seidenberg M, Durgerian S, Antuono P, et al. Neurolmage interactive effects of physical activity and APOE- $\varepsilon 4$ on BOLD semantic memory activation in healthy elders. Neuroimage. 2011;54(1): 635-44 Available from: https://doi.org/10.1016/j.neuroimage.2010.07.070

48. Kerestes R, Phal PM, Steward C, Moffat BA, Salinas S, Cox KL, et al. Alterations in dorsal and ventral posterior cingulate connectivity in APOE $\varepsilon$ 4 carriers at risk of Alzheimer's disease. BJPsych Open. 2015;1:139-48.

49. Tolppanen A-M, Solomon A, Kulmala J, Kareholt I, Ngandu T, Rusanen M, et al. Leisure-time physical activity from mid- to late life, body mass index and risk of dementia. Alzheimers Dement. 2015;11:434-43.

50. Fenesi B, Fang H, Kovacevic A, Oremus M, Raina P, Heisz J. Physical exercise moderates the relationship of apolipoprotein E (APOE) genotype and dementia risk: a population-based study. J Alzheimers Dis. 2017;56(1):297-303.

51. Yang S, Weng P, Chen J, Chiou J, Lew-ting C, Chen T, et al. ScienceDirect Leisure activities, apolipoprotein E e4 status, and the risk of dementia. J Formos Med Assoc. 2015;114(12):1216-24 Available from: https://doi.org/10. 1016/j.jfma.2014.09.006.

52. Shih I, Paul K, Haan M, Yu Y, Ritz B. Physical activity modifies the influence of apolipoprotein E $\varepsilon 4$ allele and type 2 diabetes on dementia and cognitive impairment among older Mexican Americans. Alzheimers Dement. 2018;14:1-9.

53. Jessen F, Amariglio RE, Van Boxtel M, Breteler M, Dubois B, Dufouil C, et al. A conceptual framework for research on subjective cognitive decline in preclinical Alzheimer's disease. Alzheimers Dement. 2014;10:844-52.

54. Gallagher D, Coen R, Kilroy D, Belinski K, Bruce I, Coakley D, et al. Anxiety and behavioural disturbance as markers of prodromal Alzheimer's disease in patients with mild cognitive impairment. Geriatr Psychiatry. 2010;26(2):166-72.

55. Mah L, Binns MA, Steffens DC. Alzheimer's disease neuroimaging initiative. Geriatr Psychiatry. 2015;23(5):466-76 HHS Public Access.

56. Raichlen DA, Alexander GE. Adaptive capacity: an evolutionary neuroscience model linking exercise, cognition, and brain Health. Trends Neurosci. 2017; 40(7):408-21 Available from: https://doi.org/10.1016/j.tins.2017.05.001.

57. Kempermann G, Fabel K, Ehninger D, Babu H, Leal-galicia P, Garthe A, et al. Why and how physical activity promotes experience-induced brain plasticity. Front Neurosci. 2010;4(December):1-9.

58. Kennedy G, Hardman RJ, Macpherson H, Scholey AB, Pipingas A. How does exercise reduce the rate of age-associated cognitive decline? A review of potential mechanisms. J Alzheimers Dis. 2017:55:1-18.

59. Phillips C, Baktir MA, Srivatsan M, Salehi A. Neuroprotective effects of physical activity on the brain: a closer look at trophic factor signaling. Front Cell Neurosci. 2014;8(June):1-16 Available from: http://journal.frontiersin.org/ article/10.3389/fncel.2014.00170/abstract.

60. Lodge M, Bischofberger J. Synaptic properties of newly generated granule cells support sparse coding in the adult hippocampus. Behav Brain Res. 2019;372(April). Available from: https://doi.org/10.1016/j.bbr.2019.112036.

61. Raichlen DA, Alexander GE. Exercise, APOE genotype, and the evolution of the human lifespan. Trends Neurosci. 2014:37(5):247-55 Available from: https://doi.org/10.1016/j.tins.2014.03.001.

\section{Publisher's Note}

Springer Nature remains neutral with regard to jurisdictional claims in published maps and institutional affiliations. 